\title{
Surgical management of cervical cord injury with ossification of the cervical posterior longitudinal ligament
}

\author{
Da-long Yang MD, Ying-ze Zhang MD, Yong Shen MD, Jun-ming Cao MD \\ Department of Spinal Surgery, The Third Affiliated Hospital of Hebei Medical University, Shijiazhuang, China
}

Objective: To identify an appropriate surgical approach for the management of cervical cord injury with ossification of the posterior longitudinal ligament.

Methods: A retrospective study of 25 cases of cervical cord injury with ossification of the posterior longitudinal ligament was performed. Two cases were classified as Frankel grade A, three as grade B, fourteen as grade C, and six as grade D. Treatment procedures consisted of anterior decompression with instrumentation (twelve patients), posterior decompression (eight patients), and combined anterior and posterior decompression (five patients).

Results: There were no iatrogenic injuries of great vessels, trachea, esophagus or spinal cord. All patients were followed up for 15-86 months (average, 38.3 months). All segment with anterior fixation attained solid fusion, without implants loosening or breakage. No reclosed open-door was found after posterior laminoplasty. Twenty-one patients improved by one to two Frankel grades. The patients with complete spinal cord injury achieved no neurologic recovery, but did experience relief of upper limb pain or numbness.

Conclusion: The surgical outcomes of cervical cord injury with ossification of the posterior longitudinal ligament were satisfactory. It is important to select a suitable surgical approach according to the findings on radiological imaging and the clinical characteristics and general condition of the patients.

Key words: Cervical vertebrae; Ossification, posterior longitudinal ligament; Spinal cord, injuries; Surgical procedures, operative

\section{Introduction}

Ossification of the posterior longitudinal ligament (OPLL) is known to have a high prevalence in people of Asian races, including Japanese, Korean and Chinese. The finding of prominent OPLL does not always indicate the presence of cervical myelopathy. However, patients with OPLL sometimes present with acute cervical cord injury after minor trauma without fracture or dislocation. To date, the treatment of cervical cord injury with OPLL is controversial ${ }^{1}$. In this study, 25 cases of cervical spinal cord injury with OPLL were treated surgically and retrospectively analyzed.

Address for correspondence Yong Shen, MD, Department of Spinal Surgery, The Third Affiliated Hospital of Hebei Medical University, 139 Ziqiang, Shijiazhuang, China 050051 Tel: 0086-31188602016; Fax: 0086-311-88602316; Email: doctoryangdalong@ 163.com

Received: 11 October 2010; accepted 5 November 2010

DOI: 10.1111/j.1757-7861.2011.00131.x

\section{Materials and methods}

\section{Patients' data}

From January 2001 to October 2006, 25 patients with cervical cord injury with ossification of the cervical posterior longitudinal ligament were treated surgically in our hospital, including 16 men and 9 women with an average age of 62.3 years (range, 49-78 years). The causes of trauma were traffic accidents ( 12 cases), falls from heights ( 9 cases) and being crushed by heavy objects ( 4 cases). The patients were admitted to our hospital from 4 hours to 11 days (mean 4 days) after the trauma.

\section{Clinical and radiological assessment}

The neurological state at admission was assessed according to Frankel's classification: two patients were Grade A, three grade B, fourteen grade C, and six grade D. On admission all patients were examined with plain $\mathrm{X}$-rays (anteroposterior and lateral views), MRI scans, and CT scans of the cervical spine. Neither fractures nor dislocations were shown in any patient. The findings 


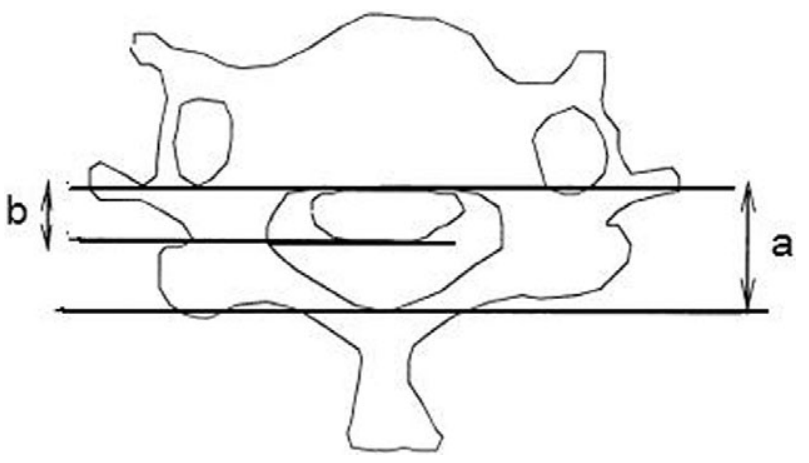

Figure 1 The occupation ratio of the ossified mass at the most stenotic level of the spinal canal is defined as follows: OPLL occupation ratio $=b / a \times 100 \%$ (a, anteroposterior diameter of the bony spinal canal; $b$, thickness of OPLL).

were continuous-type OPLL in four patients (16\%), mixed-type OPLL in five (20\%), and segmental-type OPLL in sixteen (64\%) according to the classification of Hirabayashi et al. ${ }^{2}$ The occupation ratio of the spinal canal was measured on CT scans $s^{3}$ and the ratio calculated according to the following formula:

Ratio $=($ thickness of OPLL/anteroposterior diameter of the bony spinal canal) $\times 100 \%$ (Fig. 1)

The presence of spinal cord compression and intramedullary signal changes were assessed mainly from T2-weighted MRI scans.

\section{Surgical technique}

After admission all patients received suitable first aid, timely clinical evaluation, life support and cervical restriction. Surgical treatment was performed once the patient's general condition was appropriate. One of three surgical approaches was performed according to the radiological signs.

1. Anterior approach: patients with segmental-type OPLL or continuous-type OPLL involving less than three segments and whose OPLL occupation ratio was $<50 \%$ underwent anterior cervical decompression and fusion (ACDF) (12 cases). A typical case is shown in Fig. 2. Preoperative imaging studies showed spinal cord compression at C5-6 and C6-7 with an OPLL occupation ratio $<50 \%$ (Fig. $2 \mathrm{a}-\mathrm{c}$ ), and the patient underwent anterior decompression and fusion with plate (Fig. 2d,e).

2. Posterior approach: patients with continuous-type OPLL involving more than three segments were treated by laminoplasty or laminectomy (eight cases). A typical case is shown in Fig. 3. Preoperative imaging studies showed multiple spinal cord compression
(Fig. 3a-c), and posterior decompression and enlargement plasty was performed (Fig. 3d,e).

3. Combined posterior and anterior approach: patients with segmental-type OPLL or continuous-type OPLL involving less than three segments and whose occupation ratio was $\geq 50 \%$ initially underwent laminoplasty or laminectomy followed by anterior decompression and fusion in one or two stages according to the patient's general condition (five cases). A typical case is shown in Fig. 4. Preoperative imaging studies showed spinal cord compression at the level of C3-4 with an OPLL occupation ratio $\geq 50 \%$ (Fig. $4 \mathrm{a}-\mathrm{c}$ ), and posterior decompression, enlargement plasty and complete decompression anteriorly was performed (Fig. 4d,e).

After surgery, all patients wore a soft cervical collar and were allowed to exercise within a week. Antibiotics were routinely used for 5-7 days.

\section{Results}

The operations were completed successfully in all patients, the incisions healing well without wound infection. There were no severe complications such as major vessel injury, tracheobronchial damage, esophageal fistula or spinal cord deterioration. The patients were followed up for 15-86 months with an average of 38.3 months. With regard to surgical complications, among the patients who had undergone ACDF, solid fusion was achieved in all patients by 6 months after surgery and no device-related complications were observed. In patients with laminoplasty, no door re-closer or cervical deformity was observed. Neurological grade (Frankel grade) was improved in $21(84 \%)$ of 25 patients, 14 cases increased by one level and 7 cases increased by two level (Table 1). Slight improvement in the level of sensory loss was obtained after surgery in the four cases without neurological improvement.

Table 1 Frankel grade before and after surgery

\begin{tabular}{lrrrrrr}
\hline \hline & & \multicolumn{5}{c}{ Postoperation } \\
\cline { 3 - 7 } Preoperation & No. & A & B & C & D & E \\
\hline A & 2 & 1 & 1 & - & - & - \\
B & 3 & - & 1 & 1 & 1 & - \\
C & 14 & - & - & 2 & 6 & 6 \\
D & 6 & - & - & - & - & 6 \\
E & 0 & - & - & - & - & \\
\hline \hline
\end{tabular}



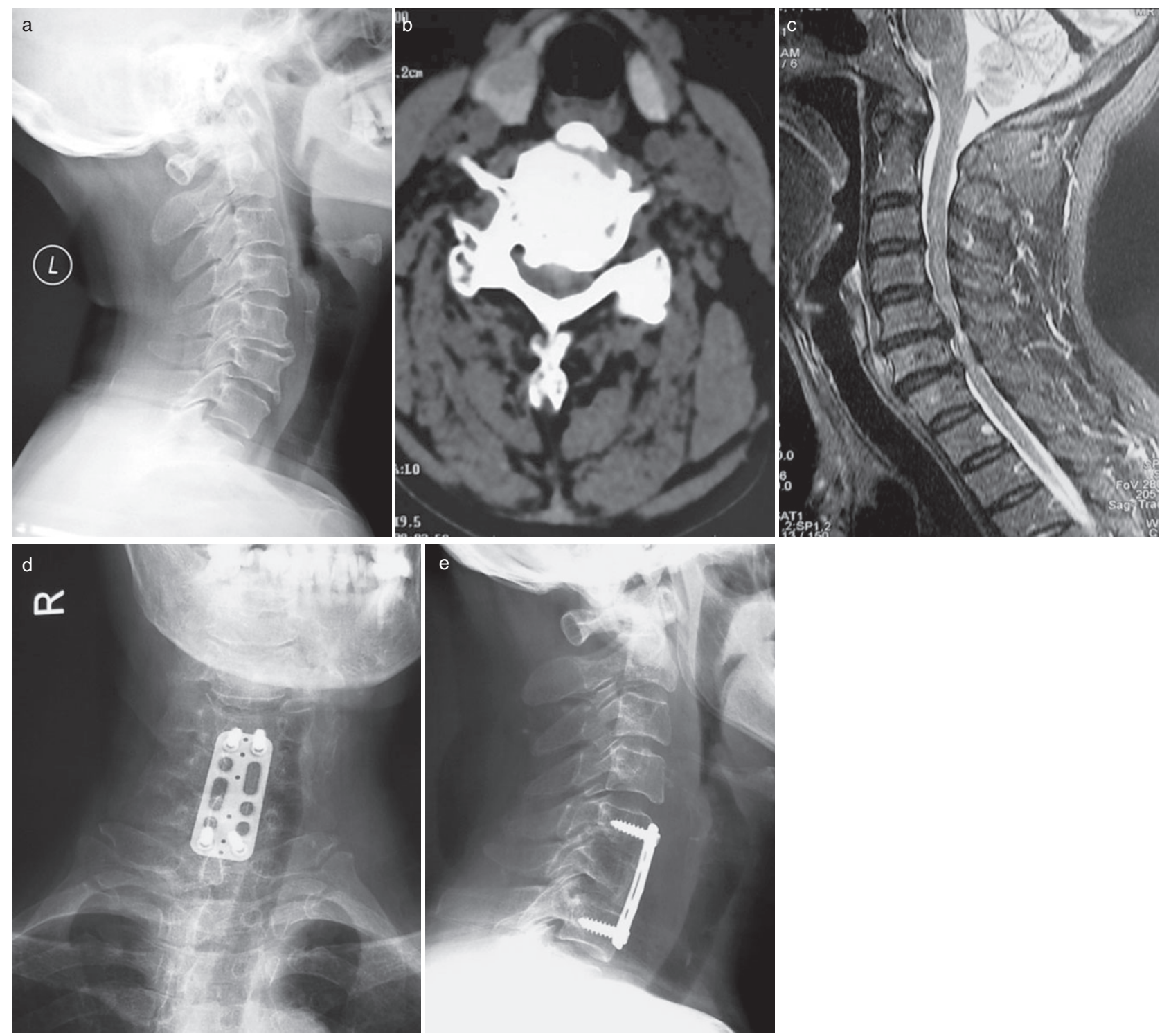

Figure 2 A 63-year-old male patient (a) preoperative lateral radiographs showing segmental OPLL at C5-6; (b) preoperative CT scans revealing OPLL occupation ratio $<50 \%$; (c) preoperative sagittal MRI demonstrating spinal cord compression at C5-6 and C6-7, a high signal intensity change at the level of the C 6 vertebral body; (d) and (e) postoperative anteroposterior and lateral radiographs showing anterior decompression and fusion with plate.

\section{Discussion}

The mechanism of cervical spinal cord injury with ossification of the posterior longitudinal ligament

It has been reported that minor trauma to the neck sometimes results in deterioration of symptoms in patients with cervical OPLL ${ }^{4}$. Koyanagi et al. reported that 16 of 89 patients who sustained an acute cervical cord injury without fracture or dislocation of the spinal column were found to have OPLL ${ }^{5}$. Static compression factors are important in the development of myelopathy in cervical OPLL patients. Although patients with OPLL often have a long asymptomatic period, the spinal cord is histologically deformed with degeneration of neurons, making it susceptible to further devastating deterioration after relatively minor trauma to the cervical spine. Most patients in our series had not exhibited cervical myelopathy before injury in spite of having narrowed spinal canals because of OPLL. The sagittal diameter of the spinal canal has been shown to be a major factor in the relationship between cervical spondylosis and symptomatic myelopathy $y^{6}$. OPLL is the most common cause of cervical spinal 

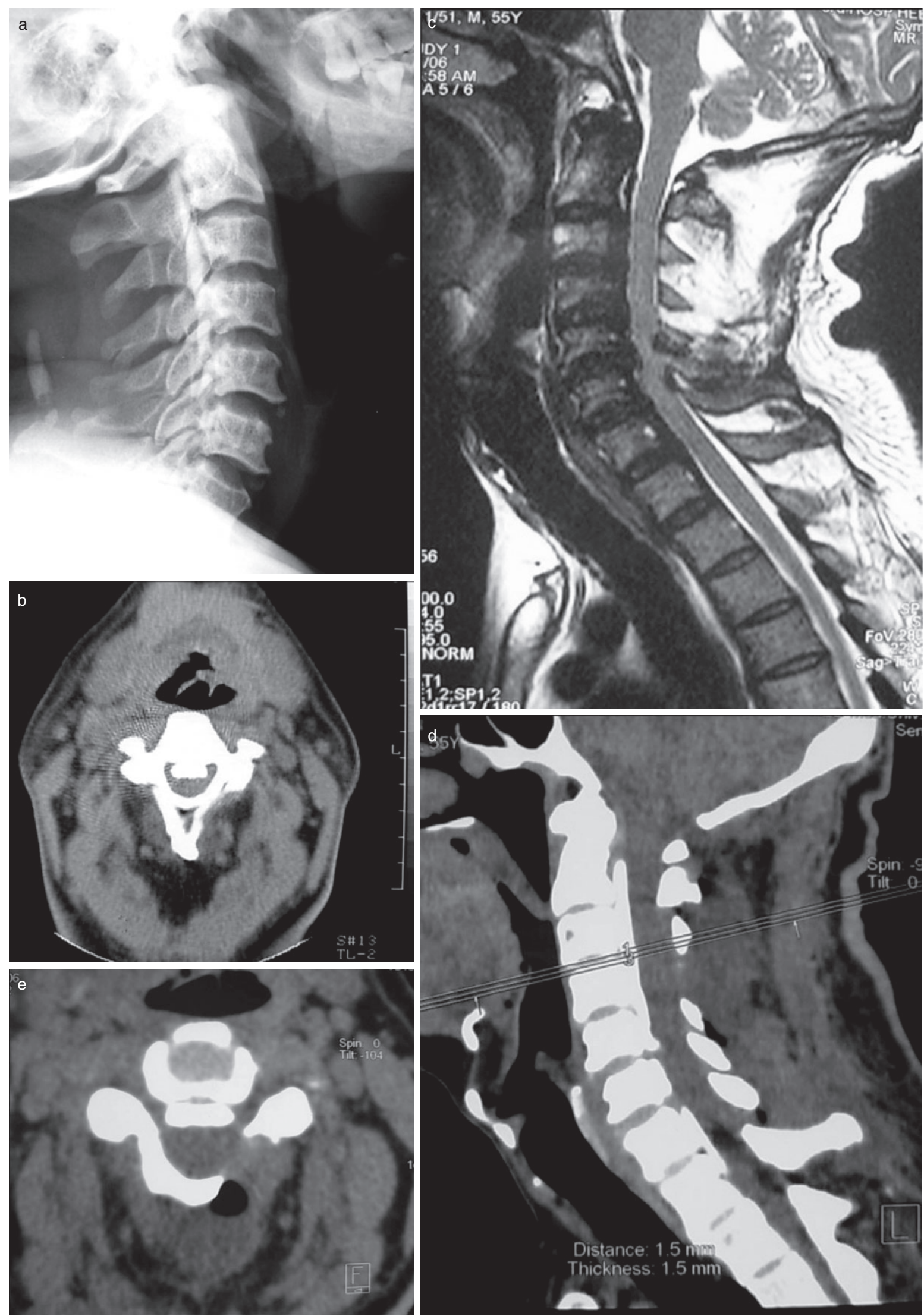

Figure 3 A 66-year-old female patient (a) preoperative lateral radiographs showing continuous-type OPLL at C2-C5; (b) preoperative CT scans revealing OPLL occupation ratio $<50 \%$; (c) preoperative sagittal MRI demonstrating multiple points of spinal cord compression; (d) and (e) postoperative CT scan revealing cervical vertebral canal posterior decompression and enlargement plasty. 

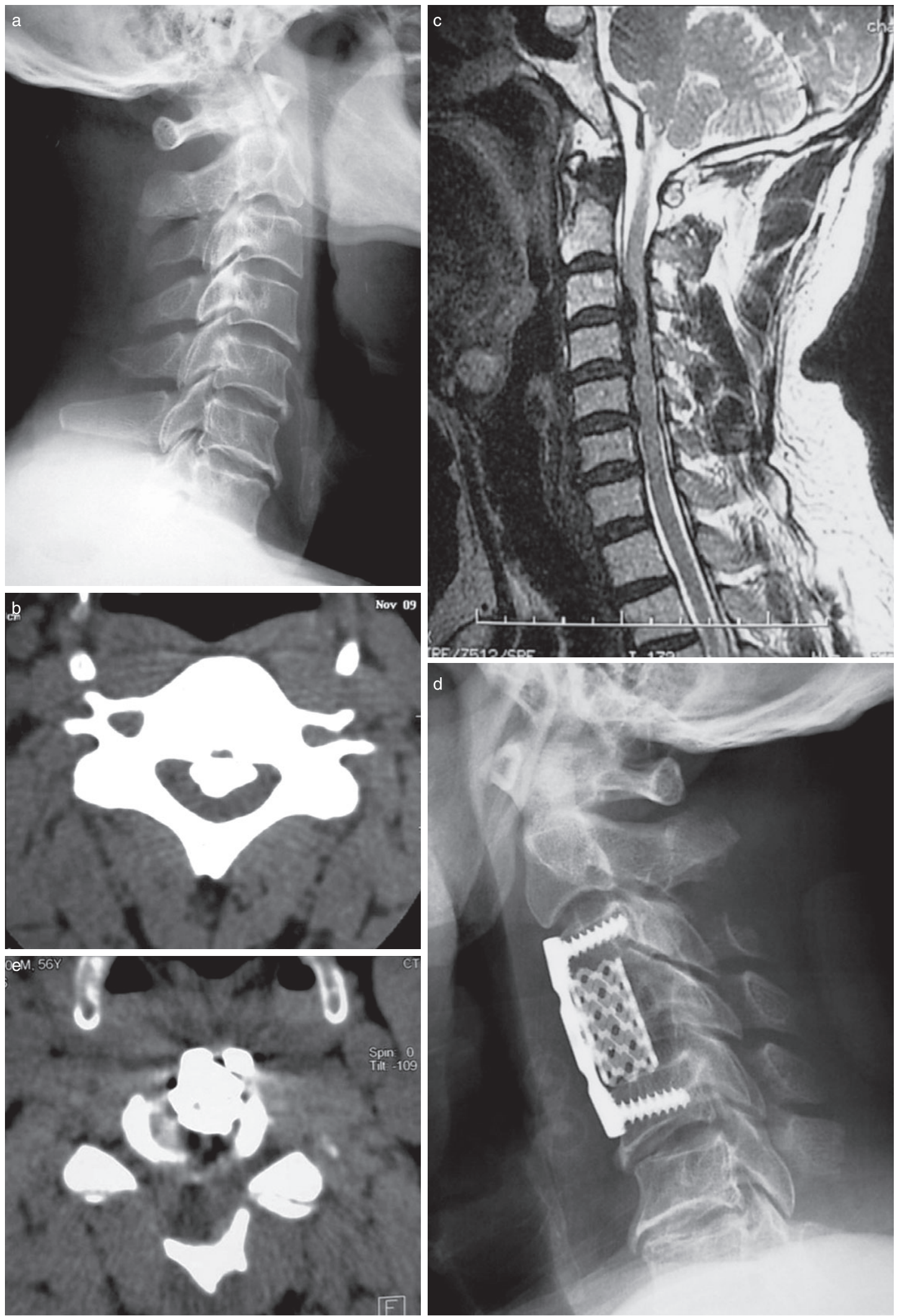

Figure 4 A 52-year-old male patient (a) preoperative lateral radiographs showing segmental OPLL at C3-4; (b) preoperative CT scans revealing OPLL occupation ratio $>50 \%$; (c) preoperative sagittal MRI demonstrating severe spinal cord compression, a high signal intensity change at the level of C3-4; (d) postoperative lateral radiographs showing anterior decompression and fusion with a plate and titanium; (e) postoperative CT scan revealing cervical vertebral canal posterior decompression and enlargement plasty and a complete decompression anteriorly. 
stenosis. Under normal circumstances, there is a "cushion space" in the spinal canal that provides room for some swelling of the spinal cord. The mechanism of injury is thought to be hyperextension of the cervical spine, followed by compression of the spinal cord between the OPLL and ligamentum flavum in the already stenotic spinal canal, which compromises the central gray matter of the spinal cord. On the other hand, OPLL creates local fixation that leads to movement of adjacent segments increasing in compensation. Trauma causing abrupt movement, usually hyperextension, between the ossified and adjacent non-ossified regions can result in acute spinal cord injury.

\section{Indications for surgical treatment}

Surgical treatment of patients with OPLL presenting with acute cervical cord injury is controversial. According to a retrospective study by Endo et al. of 15 patients with spinal cord injury and OPLL, there was no difference between the patients treated conservatively and those treated surgically in neurological improvement ${ }^{7}$. However, a prospective study by Chen et al. of 37 patients with cervical spondylosis with incomplete cervical cord injury revealed that the surgically treated group displayed statistically significant improvement at 1 and 6 months after trauma compared with the conservative treatment group ${ }^{8}$. In our series, neurological grade (Frankel grade) was improved in $21(84 \%)$ of 25 patients after surgery. Although neurological improvement can be achieved by conservative treatment, the pathology of the damaged spinal cord cannot be changed by it. Long term compression of the spinal cord may lead to ischemia, anoxia and deterioration. Prevention or reduction of secondary damage is the major objective in managing spinal cord injury. Early decompression can lessen the pressure on the spinal cord and improve the microcirculation, which in turn provides a favorable external environment for the recovery of the spinal cord. Most authors believe that surgical treatment is the preferred option for cervical cord injury without fracture or dislocation ${ }^{9,10}$.

\section{Surgical strategy for cervical cord injury with ossification of the posterior longitudinal ligament}

Imaging findings play a crucial role in surgical planning. Where OPLL affects three or more vertebral levels, posterior surgery is preferred. The basic principle of posterior surgery is to widen the spinal canal without removing the ossified mass, and includes laminectomy and expansive open door laminoplasty. Laminoplasty, being less difficult than an anterior approach and preserving the posterior structure of the cervical spine compared with laminectomy, is believed to produce better results in patients with cervical spinal stenosis. However, Iwasaki et al. have reported that the clinical outcome after laminoplasty is unsatisfactory in patients with an OPLL occupation ratio of $>50 \%{ }^{11}$. A contributing factor may be that the OPLL remains ventral to the spinal cord even after surgery. Ossification is a progressive disease, and it has been reported that the area of ossification increases after decompressive surgery ${ }^{12}$.

As OPLL is located anterior to the cord, anterior removal of OPLL is theoretically the best approach for surgical intervention. Additionally, after a solid spinal fusion, pressure on the injured spinal cord can be relieved. The anterior approach allows removal of the offending ossified ligament. Technically, it is an involved procedure, including a multilevel vertebrectomy, removal of osteophytes and OPLL, and interbody fusion with bone graft and metal plate fixation. Masaki et al. have demonstrated that the surgical outcome of ACDF is superior to the surgical outcome of laminoplasty ${ }^{13}$. However, more skillful techniques are required for ACDF than for laminoplasty. When the OPLL occupation ratio is $>50 \%$, the space available for the spinal cord is limited. With severe cervical canal stenosis, a direct anterior approach is very dangerous and may produce further cord damage ${ }^{14}$. We performed a combined posterior and anterior approach ${ }^{15}$. Posterior decompression is carried out first, followed by anterior removal of the OPLL. Posterior decompression can provide more space for cord movement, which can provide room for the subsequent anterior surgery and reduce the pressure inside the vertebral canal. Anterior decompression with removal of massive OPLL can be undertaken immediately, or 7-10 days after the posterior approach, according to the patient's general conditions. Anterior procedures can vary because of insufficient decompression resulting from ossification of the dura or massive bleeding from the epidural space. The anterior floating method in which the spinal cord is decompressed without total resection of the ossified lesion may be a good choice. We recommend the following surgical protocol for cervical cord injury with cervical OPLL based on the radiological patterns of compression: (i) patients with segmental-type OPLL or continuous-type OPLL involving less than three segments whose occupation ratio is $<50 \%$ should undergo ACDF; (ii) patients with continuous-type OPLL involving more than three levels, or who are too old or infirm to tolerate anterior surgery should undergo laminoplasty or laminectomy; and (iii) patients with segmental-type OPLL or continuous-type OPLL involving less than three segments whose occupation ratio is $>50 \%$ should firstly undergo laminoplasty or laminectomy, followed by anterior decompression and fusion in one or two stages according to the patient's general condition. 


\section{Disclosure}

No benefits in any form have been, or will be, received from a commercial party related directly or indirectly to the subject of this manuscript.

\section{References}

1. Koyanagi I, Iwasaki $Y$, Hida K, et al. Acute cervical cord injury associated with ossification of the posterior longitudinal ligament. Neurosurgery, 2003, 53: 887891.

2. Hirabayashi K, Miyakawa J, Satomi K, et al. Operative results and postoperative progression of ossification among patients with ossification of cervical posterior longitudinal ligament. Spine, 1981, 6: 354-364.

3. Kato $Y$, Iwasaki M, Fuji T, et al. Long-term follow-up results of laminectomy for cervical myelopathy caused by ossification of the posterior longitudinal ligament. J Neurosurg, 1998, 89: 217-223.

4. Eismont FJ, Clifford S, Goldberg M, et al. Cervical sagittal spinal canal size in spine injury. Spine, 1984, 9: 663-666.

5. Koyanagi I, Iwasaki Y, Hida K, et al. Acute cervical cord injury without fracture or dislocation of the spinal column. J Neurosurg, 2000, 93 (Suppl.): S15-S20.

6. Katoh $\mathrm{S}$, Ikata $\mathrm{T}$, Hirai $\mathrm{N}$, et al. Influence of minor trauma to the neck on the neurological outcome in patients with ossification of the posterior longitudinal ligament (OPLL) of the cervical spine. Paraplegia, 1995, 33: 330-333.

7. Endo $\mathrm{S}$, Shimamura $\mathrm{T}$, Nakae $\mathrm{H}$, et al. Cervical spinal cord injury associated with ossification of the posterior longitudinal ligament. Arch Orthop Trauma Surg, 1994, 113: 218-221.
8. Chen TY, Dickman CA, Eleraky $M$, et al. The role of decompression for acute incomplete cervical spinal cord injury in cervical spondylosis. Spine, 1998, 23: 2398-2403.

9. Yang JS, Huang WD, Wang SB. Comparative observation on operative and non-operative effects in treatment of cervical spinal cord injury without fracture dislocation (Chin). Zhonghua. Chuang Shang Za Zhi, 2004, 20: 330-332.

10. Li $W$, Lin $Z X, C$ hen $Y$, et al. Operative treatment of cervical spinal cord injury without fracture or dislocation (Chin). Zhonghua. Chuang Shang Za Zhi, 2005, 21: 499-501.

11. Iwasaki M, Okuda S, Miyauchi A, et al. Surgical strategy for cervical myelopathy due to ossification of the posterior longitudinal ligament: Part 1: clinical results and limitations of laminoplasty. Spine, 2007, 32: 647-653.

12. Kawaguchi $Y$, Kanamori $M$, Ishihara $H$, et al. Progression of ossification of the posterior longitudinal ligament following en bloc cervical laminoplasty. J Bone Joint Surg Am, 2001, 83: 1798-1802.

13. Masaki $Y$, Yamazaki M, Okawa $A$, et al. An analysis of factors causing poor surgical outcome in patients with cervical myelopathy due to ossification of the posterior longitudinal ligament: anterior decompression with spinal fusion versus laminoplasty. J Spinal Disord Tech, 2007, 20: 7-13.

14. Xu ZW, Zhang JX. One stage anteroposterior decompression and internal fixation for ossification of posterior longitudinal ligament (Chin). Zhonghua. Chuang Shang Za Zhi, 2005, 21: 595-597.

15. Lv ZM, Shen $Y, X u Y J$, et al. Posterior-anterior approach surgical treatment for severe cervical spondylotic myelopathy (Chin). Zhongguo Jiao Xing Wai Ke Za Zhi, 2007, 15: 344-346. 